\title{
The Selection of Proper Auxiliary Parameters in the Homotopy Analysis Method. A Case Study: Uniform Solutions of Undamped Duffing Equation
}

\author{
Mustafa Turkyilmazoglu \\ Mathematics Department, University of Hacettepe, Ankara, Turkey \\ E-mail: turkyilm@hotmail.com \\ Received March 20, 2011; revised May 9, 2011; accepted May 12, 2011
}

\begin{abstract}
The present paper is concerned with two novel approximate analytic solutions of the undamped Duffing equation. Instead of the traditional perturbation or asymptotic methods, a homotopy technique is employed, which does not require a small perturbation parameter or a large parameter for an asymptotic expansion. It is shown that proper choices of an auxiliary linear operator and also an initial approximation during the implementation of the homotopy analysis method can yield uniformly valid and accurate solutions. The obtained explicit analytical expressions for the solution predict the displacement, frequency and period of the oscillations much more accurate than the previously known asymptotic or perturbation formulas.
\end{abstract}

Keywords: Homotopy Analysis Method, Auxiliary Linear Operator, Initial Approximation, Duffing Oscillator, Uniform Solution

\section{Introduction}

The importance of nonlinear Duffing equation has been widely understood among the scientists since it plays a key role in some important practical phenomena such as periodic orbit extraction, non uniformity caused by an infinite domain, nonlinear mechanical oscillators, prediction of diseases, and so on.

Since the Duffing equation engulfs a strong nonlinearity within itself, it's full analytical solution has not been achieved so far. Therefore, considerable attention has been directed towards the study of strongly nonlinear oscillators and several methods have been used to find approximate solutions to nonlinear oscillators. Most of the methodologies involve the introduction of a small parameter into the equation, which might be artificial, and then expansion of the solution through the perturbation series around this parameter. However, the solutions obtained within this approach may not be uniform, restricting the applicability of such perturbation methods $[1,2]$. To overcome the limitations of the perturbative techniques, many novel techniques have been proposed in recent years. For example, modified Lindstedt-Poincare method $[3,4]$ and variational iteration methods [5]. Some other numerical and approximate methods follow [6-10].

Liao in [11] proposed a new technique which is based on the homotopy concept in topology, named the homotopy analysis method (HAM). Unlike the aforementioned traditional perturbation methods, this technique does not require a small perturbation parameter in the equation. In this method, according to the homotopy technique, a homotopy with an imbedding parameter is constructed, and the imbedding parameter is considered as a small parameter. Thus the original nonlinear problem is converted into an infinite number of linear problems without using the perturbation techniques, see the book by Liao [12]. Different from other methods, the HAM provides a simple way to control and adjust the convergence region of solution series by means of an auxiliary parameter $[13,14]$.

Following the introduction of HAM by Liao, He in [15] proposed the so-called homotopy perturbation method (HPM). Unfortunately, Sajid and Hayat [16] pointed out that the so-called homotopy perturbation method has nothing new except its new name, because the HPM is only a special case of the homotopy analysis method (HAM) when so that all results given by the HPM can be obtained by the HAM as a special case. Moreover, as a special case of the HAM, the HPM can not give con- 
vergent series solution for strongly nonlinear problems. Abbasbandy in [17] gave a simple example to show that, like perturbation approximations, the results given by the HPM are divergent when the physical parameters become large. Among many other authors VanGorder and Vajravelu in [18] also pointed out this fact. More recently, Liang and Jeffrey in [19] pointed out that in some cases the series solution given by the HPM and VIM (another method proposed also by $\mathrm{He}$ in [20]) is divergent at all points except $t=0$ that however defines the initial condition.

We in the present paper use the homotopy analysis technique for the solution of the nonlinear Duffing equation. Better auxiliary linear operators and initial approximations are the essential target to be used here within the homotopy concept so that highly accurate solutions have been obtained. The proposed linear operators together with the homotopy analysis method provide formulas for the displacement, frequency and period of the oscillations, which are valid for all the existing parameters and also are more accurate than those already available in the literature.

The following strategy is adopted in the rest of the paper. In Section 2 the idea of homotopy analysis method is laid out. Application of the method is implemented in Section 3, in which, first the homotopy analysis method is revisited and later using different auxiliary parameters and initial approximations, analytic expressions are derived yielding better results. Section4 presents compari- sons of the period and displacement between those in [15], our findings and the exact numerical ones. Finally conclusions follow in Section 5.

\section{The Homotopy Analysis Method}

Liao in [1] proposed a new kind of analytic technique for nonlinear problems, namely the homotopy analysis method. This method is based on the homotopy and has several advantages. To underline, firstly its validity does not depend upon whether or not nonlinear equations under consideration contain small or large parameters, hence it can solve more of strongly nonlinear equations than the perturbation techniques. Secondly, it provides us with a great freedom to select proper auxiliary linear operators and initial guesses so that uniformly valid approximations can be obtained. Thirdly, it gives a family of approximations which are convergent in a larger region. Liao successfully applied the homotopy analysis method to solve some nonlinear problems in mechanics. For example, Liao in [21] gave a purely analytic solution of 2D Blasius's viscous flow over a semi-infinite flat plate, which is uniformly valid in the whole physical region. Further examples are provided within the reference [12]. To briefly describe the method let's consider the following nonlinear differential equation

$$
N(u)-f(r)=0, r \in \Omega
$$

with boundary conditions

$$
B\left(u, \frac{\partial u}{\partial n}\right)=0, r \in \Gamma
$$

where $N$ is a nonlinear operator, $B$ is a boundary operator, $u$ is an unknown analytic function and $\Gamma$ is the boundary of the domain $\Omega$. By this technique, we construct a homotopy $v(r, p)$ from the cartesian set $\Omega \times[0,1]$ to $R$ which satisfies

$$
H(v, p)=(1-p) L\left(v-u_{0}\right)+p h[N(u)-f(r)]=0,(3)
$$

where $p \in[0,1]$ is an embedding parameter, $u_{0}$ is an initial approximation of Equation (1) that satisfies the boundary condition (2) and $h$ is a constant that can be adjusted to speed the convergence. It is clear from Equation (3) that for $p=0$ and $p=1$ respectively the followings hold

$$
\begin{aligned}
& H(v, 0)=L\left(v-u_{0}\right)=0, \\
& H(v, 1)=N(u)-f(r)=0 .
\end{aligned}
$$

Hence, it can be deduced from (4) that the deformation process of $p$ from zero to unity is just that of the solution from $u_{0}(r)$ to $u(r)$. Further, considering $p$ as a parameter, the solution to system (1-2) can be naturally expressed taking into account a Taylor expansion of the solution $v(t, p)$ at $p=0$ and later imposing the expansion at $p=1$

$$
u(t)=u_{0}(t)+\sum_{k=1}^{\infty} u_{k}(t)
$$

where $u_{k}$ are defined by $u_{k}=\left.\frac{1}{k !} \frac{\partial u}{\partial p}\right|_{p=0}$.

\section{Application to Duffing Equation}

Duffing equation describes many kinds of nonlinear oscillatory systems in physics, mechanics and engineering. The unforced, undamped Duffing equation that we consider consists of the nonlinear initial value system

$$
\frac{\mathrm{d}^{2} u}{\mathrm{~d} t^{2}}+u+\varepsilon u^{3}=0, u(0)=A, u^{\prime}(0)=0 .
$$

The parameters $\varepsilon$ and $A$ in (6) measure respectively the nonlinearity and amplitude of the displacement $u(t)$. Actually the period of the solution can be formulated analytically as in [2]

$$
T=\frac{4}{\sqrt{1+\varepsilon A^{2}}} \int_{0}^{\frac{\pi}{2}} \frac{\mathrm{d} x}{\sqrt{1-k \sin ^{2} x}}, \quad k=\frac{\varepsilon A^{2}}{2\left(1+\varepsilon A^{2}\right)} .
$$


For the system (6) Liao in [22] suggested the subsequent auxiliary linear operator

$$
L(u)=\frac{\mathrm{d}^{2} u}{\mathrm{~d} t^{2}}+u
$$

with an assumption of the initial solution of the form

$$
u_{0}=A \cos \alpha t
$$

such that $\alpha(\varepsilon)$ is a nonzero unknown constant with the property of $\alpha(0)=1$. Considering the homotopy introduced in (3) with $h=1$ and imposing the constraint that no secular terms are allowed, He in [15] obtained a first-order approximation to the solution in subsequent form

$$
u=\frac{3 \varepsilon A^{2}}{4\left(\alpha^{2}-1\right)} \cos \alpha t+\frac{\varepsilon A^{3}}{4\left(9 \alpha^{2}-1\right)} \cos 3 \alpha t
$$

in which $\alpha$ is defined as

$$
\alpha=\frac{\sqrt{10+7 \varepsilon A^{2}+\sqrt{64+104 \varepsilon A^{2}+49 \varepsilon^{2} A^{4}}}}{18} .
$$

It should be remarked here that the solution given in (10) has a non removable singularity for $\alpha= \pm 1$.

Carrying out a further step, it is easy to obtain the next-order solution $u_{2}$ in (5) which can later be combined with (10) to form a second-order solution. He then obtained the period of the approximate solution by

$$
T=\frac{2 \pi}{\alpha},
$$

with $\alpha$ given as in (11). He, later claimed that the solution he achieved by the homotopy technique with the properly chosen auxiliary parameters is of high accuracy and it is valid for a very large values of $\varepsilon$. Denoting the ratio of exact period (7) to approximate one (12) in the limit of $\varepsilon$ tending to infinity as rat, He found that rat $=1.04096$ (it should be emphasized that a simple error occurred in [15] for the evaluation of rat). Therefore, He in [15] concluded that for any value of $\varepsilon$ the maximal relative error is only 4 percent.

In place of the linear operator (8), He [23] proposed to use

$$
L(u)=\frac{\mathrm{d}^{2} u}{\mathrm{~d} t^{2}}+\alpha^{2} u
$$

with the same assumption of the initial solution as in (9) and $h=1$. If the occurrence of secular terms in the solution is eliminated in the first iteration, the solution to Duffing Equation (6) can be written at the first-order in the form

$$
u=A \cos \alpha t-\frac{\varepsilon A^{3} \sin \alpha t \sin 2 \alpha t}{16 \alpha^{2}}
$$

with $\alpha$ defined as

$$
\alpha=\frac{1}{2} \sqrt{4+3 A^{2} \varepsilon} .
$$

It can be immediately seen that the displacement $u$ in (14) does not blow up for any physical value of $\alpha$ and the form of $\alpha$ obtained in (15) is much simpler than Equation (11) obtained in [15]. With this frequency, the ratio between the exact and approximate period can be computed as 1.0222 in the limit $\varepsilon \rightarrow \infty$. Thus (15) represents a better estimate and only 2 percent relative error occurs, even for infinite value of $\varepsilon$. A comparison between (10-11) and (14-15) will be performed later in section Section 4.

It is a well-known fact that in the homotopy method there is a great freedom for the choice of auxiliary linear operator and initial approximate solution. We prove, in what follows, that with different proper selections, a better solution than (10-11) can be obtained.

\subsection{A New Proposed Homotopy 1}

Making a substitution $\tau=\omega t$ permits the frequency $\omega$ to appear explicitly in the Duffing Equation (6). This is actually a coupled technogy of homotopy analysis method with the Lindstedt Poincare method. Afterwards, keeping the linear operator as in (8) with $t$ replaced by $\tau$, choosing the initial approximation as $u_{0}=A \cos \tau$ with $h=\frac{1}{A^{2} \varepsilon}$ (note that this selection of $h$ is necessary to keep the consistency in the limit $\varepsilon \rightarrow \infty$ ). If the occurrence of secular terms in the solution is eliminated in the first iteration, the leading-order $\omega_{0}$ is found to be the same as (15) and the solution to the transformed Duffing equation can be written at the first-order in the form

$$
u=\frac{1}{32} A(31 \cos \tau+\cos 3 \tau) .
$$

With the frequency $\omega=\omega_{0}$, the ratio between the exact and approximate is the same as for homotopy approach of He [23] in the limit $\varepsilon \rightarrow \infty$. To get a better estimate and hence improve the frequency, we again set the coefficient of $\cos \tau$ to disappear so that secular terms in the next solution will be eliminated. This results in

$$
w_{1}=-\frac{3 A^{2} \varepsilon}{128 \sqrt{4+3 A^{2} \varepsilon}},
$$

leading to

$$
\omega=\omega_{0}+\omega_{1}=\frac{256+189 A^{2} \varepsilon}{128 \sqrt{4+3 A^{2} \varepsilon}}
$$

whose large $\varepsilon$ limit yields a ratio 1.00623 , so only 0.62 
percentage relative error is observed, which is much better than the above approaches.

\subsection{A New Proposed Homotopy 2}

If we now select the linear operator as in (13) but assume the initial approximation to the solution as $u_{0}=A \cos \omega t$, with $\omega$ denoting the frequency of the solution different from that of linear solution $\alpha$, they can be determined at the first iteration by restricting the appearance of secular terms at the next level. Within this approach $\omega$ and $\alpha$ are found to be

$$
\omega=\frac{\sqrt{\frac{-4096+4096 i \sqrt{3}+3601 i(i+\sqrt{3}) A^{4} \varepsilon^{2}+2 A^{2} \varepsilon\left(-3808+3808 i \sqrt{3}+143 \rho^{1 / 3}\right)+304 \rho^{1 / 3}-\rho^{2 / 3}-i \sqrt{3} \rho^{2 / 3}}{\rho^{1 / 3}}}}{12 \sqrt{3}},
$$

$$
\alpha=\frac{\omega \sqrt{9+7 A^{2} \varepsilon-9 \omega^{2}}}{\sqrt{1+A^{2} \varepsilon-\omega^{2}}},
$$

where

$$
\begin{aligned}
\rho= & -262144-731136 A^{2} \varepsilon-528096 A^{4} \varepsilon^{2}-59833 A^{6} \varepsilon^{3} \\
& +648 \sqrt{6} \sqrt{-A^{4} \varepsilon^{2}\left(32768+123904 A^{2} \varepsilon+166536 A^{4} \varepsilon^{2}+92513 A^{6} \varepsilon^{3}+17113 A^{8} \varepsilon^{4}\right)} .
\end{aligned}
$$

By this means, the homotopy method (3) yields the first-order solution

$$
u=A \cos \omega t+\frac{A\left(2 \cos \omega t\left(\omega^{2}\left(18+13 A^{2} \varepsilon-18 \omega^{2}\right)-\alpha^{2}\left(2+A^{2} \varepsilon-2 \omega^{2}\right)-A^{2} \varepsilon\left(\alpha^{2}-\omega^{2}\right) \cos 2 \omega t\right)\right)}{4\left(\alpha^{4}-10 \alpha^{2} \omega^{2}+9 \omega^{4}\right)} .
$$

The forms of the solution in (19) and of the frequency in (18) are more complicated when compared with those of (10-11) and (14-15). However, after a straightforward evaluation it is found that rat $=0.997314$ in the limit $\varepsilon \rightarrow \infty$, resulting in only 0.26 percent maximal relative error, which is best of all among those presented in [15] and above by our two approaches.

\section{Discussion of the Results}

In this section, to show the advantages of our homotopy approaches, a comparison is made between the full numerical simulation results of (6), those of $\mathrm{He}$ in [15] and the ones obtained in section Section 3. We particularly choose the parameters $A=1$ and $\varepsilon=1$ in what follows. For these specific values, it is straightforward to compute the correspondences of the periods, which are tabulated in Table 1. As compared to the exact period, our homotopy technique is seen to give extremely more accurate results than the homotopy perturbation employed in [15].

We next present numerical simulation results of the displacement function. Figure 1 shows the exact numerical and second-order approximate solution of $\mathrm{He}$, whereas Figures 2-4 demonstrate the exact numerical and first-order solutions from the homotopy approaches. It is observed from Figure 1 related to the second-order homotopy perturbation method of He that the uniformity of the approximate solution gets weakened as $t$ increases. On the other hand, our first-order approach in the homotopy analysis gives extremely accurate solutions when compared with the exact solution. Particularly as seen from Figure 4, a much higher accuracy can be observed from the approach 3 . Thus, the analytic approximations as displayed in Figures 2-4 are sufficiently reliable and can be used in the analysis of Duffing oscillator in further research.

It should be emphasized that only the first-order approximate solutions that we obtained in Section 3 reveal ex- cellent agreement with the exact numerical solutions. Addition of higher approximations from the homotopy technique would yield more remarkable agreement. It is furthermore worthwhile to state that the homotopy solutions obtained here are valid for all the values of the parameters $A$ and $\varepsilon$. Therefore, we do not need to approximate the small $\varepsilon$ perturbation solutions or the large $\varepsilon$ asymptotic solutions as in [2]. In fact, our

Table 1. Illustrating the periods for the Duffing Equation (6) for $A=1$ and $\varepsilon=1$. First is the exact, second is from He in [15], third is from approach of He [23], fourth is from approach 1 and the fifth is from approach 2.5

\begin{tabular}{lllll}
\hline 4.76802 & 4.73233 & 4.74964 & 4.78166 & 4.76877 \\
\hline
\end{tabular}




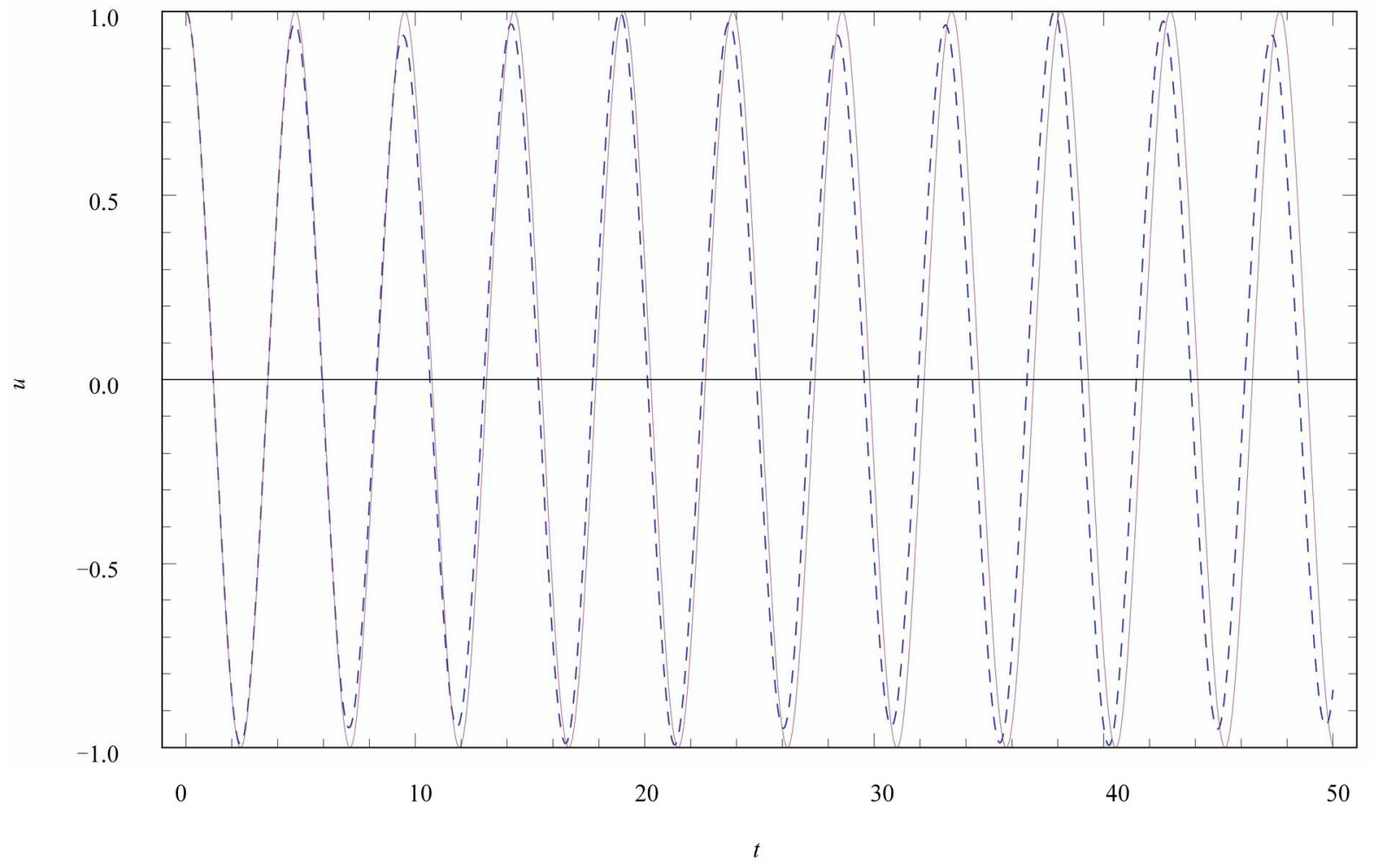

Figure 1. Solution of the Duffing Equation (6): straight curve from the numerical solution and dashed curve from the second-order solution of He (10).

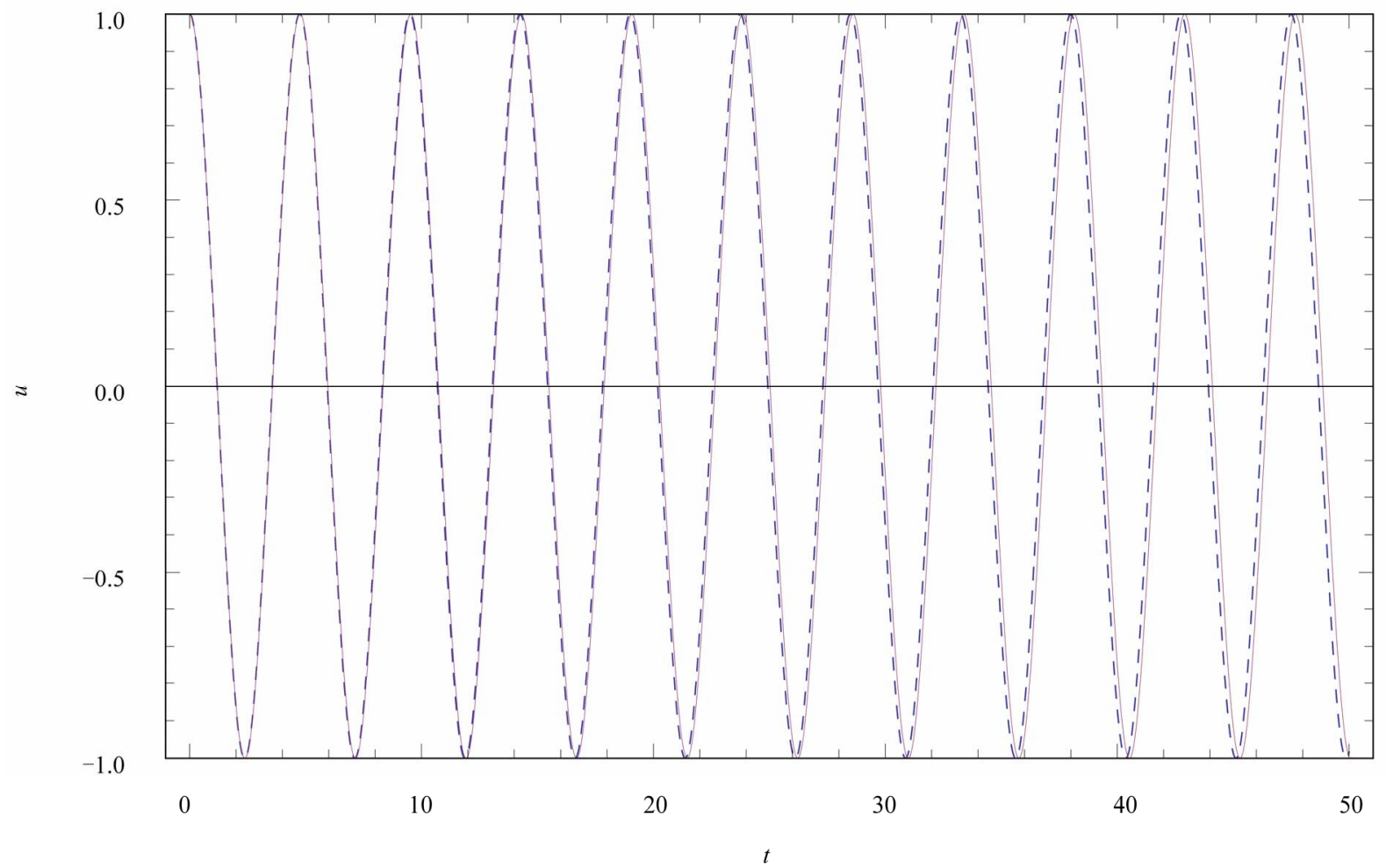

Figure 2. Solution of the Duffing Equation (6): straight curve from the numerical solution and dashed curve from the first-order solution of approach of He [23] (14). 


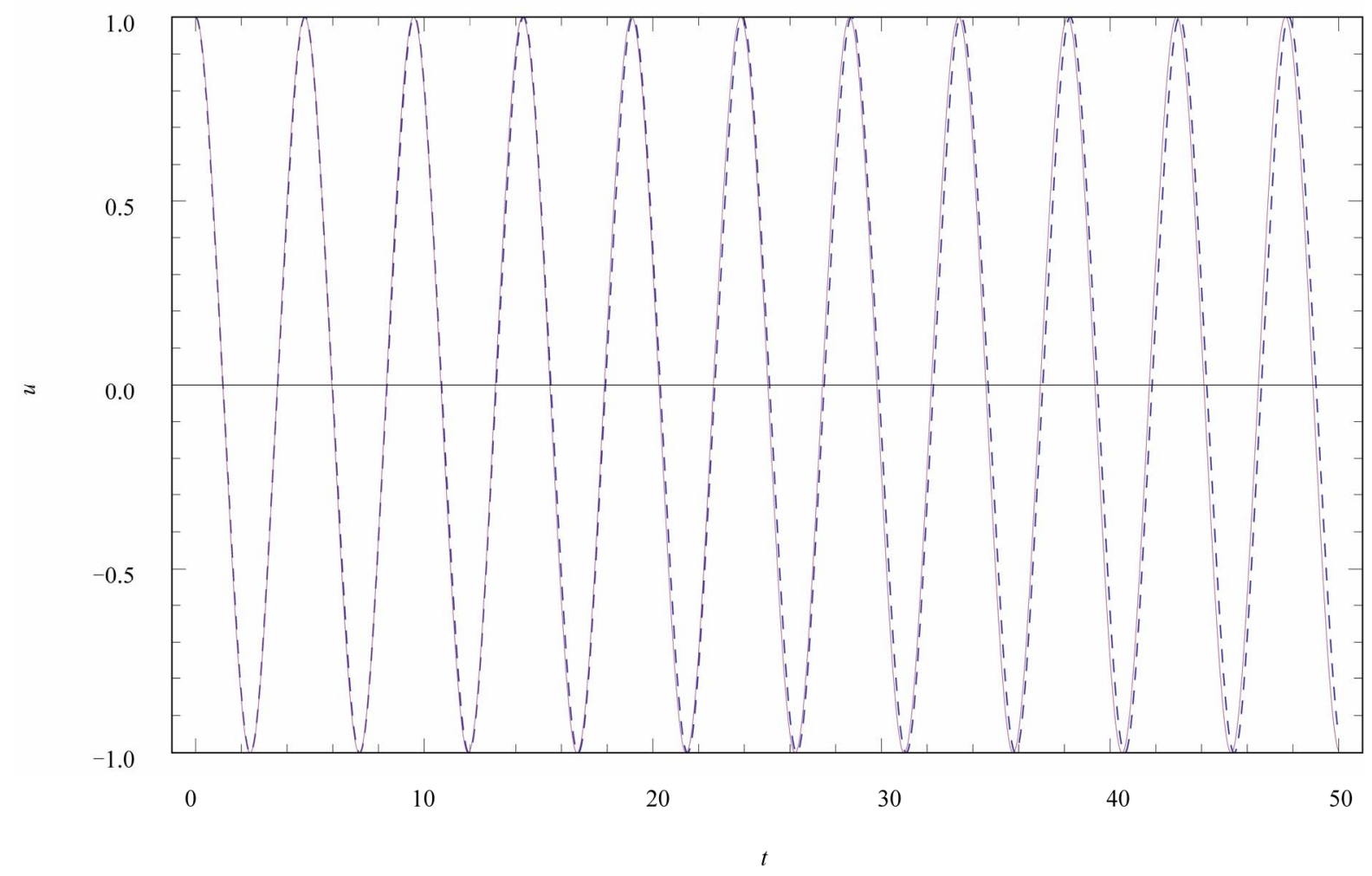

Figure 3. Solution of the Duffing Equation (6): straight curve from the numerical solution and dashed curve from the first-order solution of approach 1 (16).

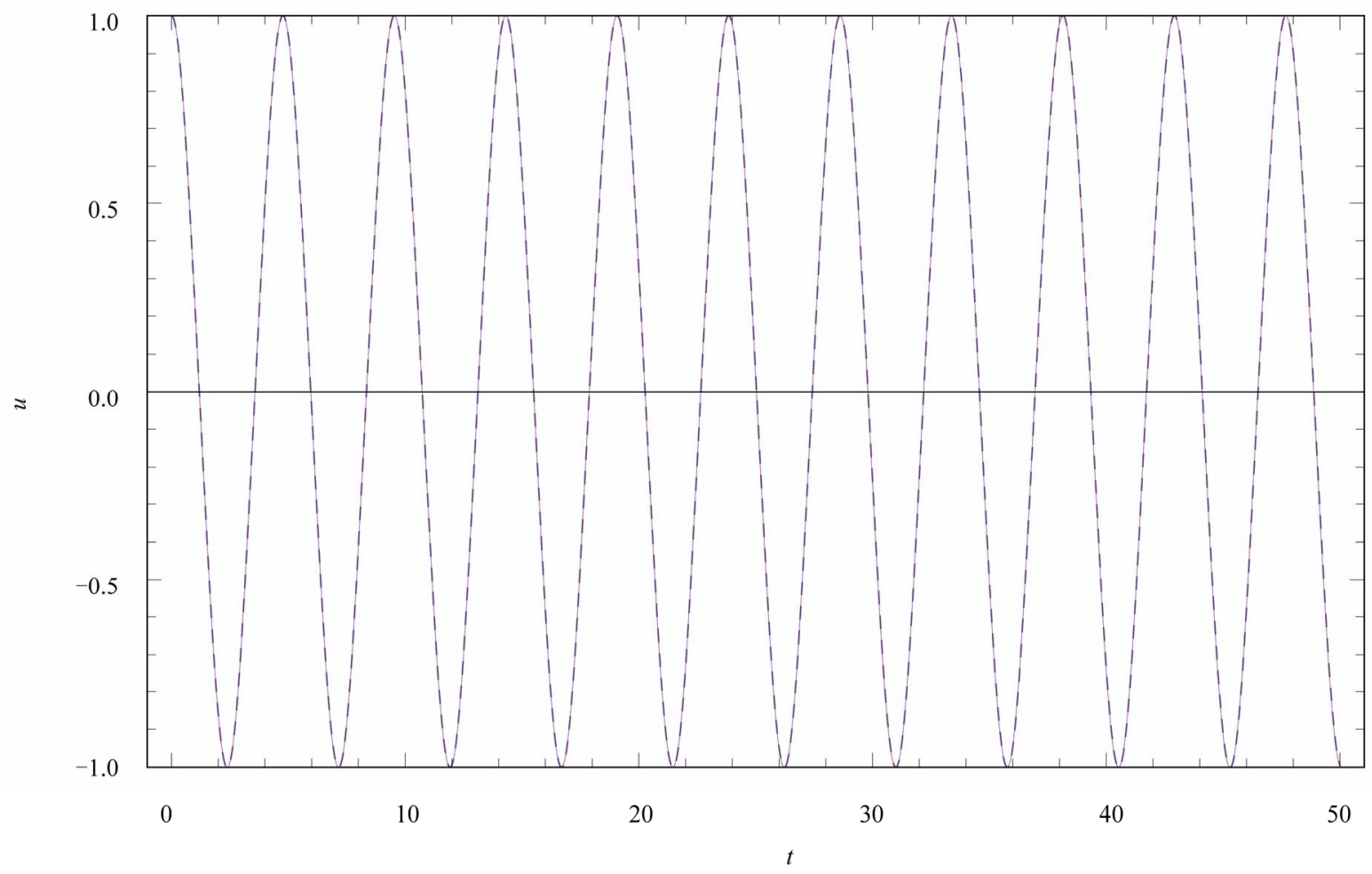

Figure 4. Solution of the Duffing Equation (6): straight curve from the numerical solution and dashed curve from the first-order solution of approach 2 (19). 
homotopy approaches are able to estimate the frequency and period of the oscillations in a better way. Moreover, unlike the solution of He, no singularity appears in the displacement solution. Thus it can be concluded that the homotopy method presented here can safely handle the situations of high nonlinearity occurring in the Duffing problems.

\section{Concluding Remarks}

In this paper the nonlinear problem of Duffing equation has been considered with the homotopy analysis technique. As compared to the perturbation methods, the homotopy treatment employed here does not require any small parameter, yielding explicit formulas for the quantities of physical desire, such as the displacement, frequency and the period of the oscillations.

Four homotopy approaches have been pursued in this analysis. First, the homotopy method of $\mathrm{He}$ in [15] has been reinvestigated further calculating the second-order contribution that was missed in [15]. Taking the advantage of free selection of the linear operator and the initial approximation to the solution, two more approaches have been proposed here. The homotopy methods with these choices are proven to generate analytic approximations that are more accurate than the result presented in [15]. Moreover, the approximate solutions obtained by these techniques are valid not only for small parameters but also very large parameters that are involved in the degree of the nonlinearity. The purely explicit analytical formulas obtained for the frequency and period agree excellently with the exact values even for infinitely large parameters, the discrepancy of the approximate period with respect to the exact one is as low as $0.26 \%$. In addition to this, the displacement function is uniformly valid and does not exhibit any singularities as compared to the solution in [15].

The homotopy technique proposed here can be safely adopted for sets of fully coupled, highly nonlinear equations governing other physical problems in science and engineering. Analytical solutions obtained here also provide a good scientific base for the validation of the numerically computed values using different schemes in the literature.

\section{References}

[1] A. H. Nayfeh and D. T. Mook, "Nonlinear Oscillations," John Willey and Sons, New York, 1979.

[2] A. H. Nayfeh, "Problems in Perturbations," John Willey and Sons, New York, 1985.

[3] Y. K. Cheung, S. H. Chen and S. L. Lau, "A Modified Lindstedt-Poincaré Method for Certain Strongly Non-Linear
Oscillators," International Journal of Non-Linear Mechanics, Vol. 26, No. 3-4, 1991, pp. 367-378.

doi:10.1016/0020-7462(91)90066-3

[4] J. I. Ramos, "On Lindstedt-Poincaré Techniques for the Quintic Duffing Equation," Applied Mathematics and Computation, Vol. 193, No. 2, 2007, pp. 303-310. doi:10.1016/j.amc.2007.03.050

[5] S. Momani and S. Abuasad, "Application of He's Variational Iteration Method to Helmholtz Equation," Chaos, Solitons \& Fractals, Vol. 27, No. 25, 2006, pp. 1119-1123. doi:10.1016/j.chaos.2005.04.113

[6] W. S. Loud, "On Periodic Solutions of Duffing's Equation with Damping," Journal of Mathematics and Physics, Vol. 34, No. 2, 1955, pp. 173-178.

[7] G. R. Liu and T. Y. Wu, "Numerical Solution for Differential Equations of Duffing-Type Non-Linearity Using the Generalized Differential Quadrature Rule," Journal of Sound and Vibration, Vol. 237, No. 5, 2000, pp. 805-817. doi:10.1006/jsvi.2000.3050

[8] D. M. Wu and Z. C. Wang, "A Mathematica Program for the Approximate Analytical Solution to a Nonlinear Undamped Duffing Equation by a New Approximate Approach," Computer Physics Communications, Vol. 174, No. 6, 2006, pp. 447-463. doi:10.1016/j.cpc.2005.09.006

[9] E. Yusufoğlu, "Numerical Solution of Duffing Equation by the Laplace Decomposition Algorithm," Applied Mathematics and Computation, Vol. 177, No. 2, 2006, pp. 572-580. doi:10.1016/j.amc.2005.07.072

[10] V. Marinca and N. Herişanu, "Periodic Solutions of Duffing Equation with Strong Non-Linearity," Chaos, Solitons \& Fractals, Vol. 37, No. 1, 2008, pp. 144-149. doi:10.1016/j.chaos.2006.08.033

[11] S. J. Liao, "The Proposed Homotopy Analysis Technique for the Solution of Nonlinear Problems," Ph.D. Thesis, Shanghai Jiao Tong University, Shanghai, 1992.

[12] S. J. Liao, "Beyond Perturbation: Introduction to Homotopy Analysis Method," Chapman \& Hall/CRC, Boca Raton-London-New York-Washington, DC, 2003. doi:10.1201/9780203491164

[13] S. J. Liao, "An Explicit, Totally Analytic Approximation of Blasius' Viscous Flow Problems," International Journal of Non-Linear Mechanics, Vol. 34, No. 4, 1999, pp. 759-778. doi:10.1016/S0020-7462(98)00056-0

[14] S. J. Liao and Y. Tan, "A General Approach to Obtain Series Solutions of Nonlinear Differential Equations," Studies in Applied Mathematics, Vol. 119, No. 4, 2007, pp. 297-254. doi:10.1111/j.1467-9590.2007.00387.x

[15] J. H. He, "An Approximate Solution Technique Depending on an Artificial Parameter: A Special Example," Communications in Nonlinear Science and Numerical Simulation, Vol. 3, No. 2, 1998, pp. 92-96. doi:10.1016/S1007-5704(98)90070-3

[16] M. Sajid and T. Hayat, "Comparison of Ham and Hpm Methods in Nonlinear Heat Conduction and Convection Equations," Nonlinear Analysis: Real World Applications, Vol. 9, No. 5, 2008, pp. 2296-2301. doi:10.1016/j.nonrwa.2007.08.007 
[17] S. Abbasbandy, "The Application of the Homotopy Analysis Method to Nonlinear Equations Arising in Heat Transfer," Physics Letter A, Vol. 360, No. 1, 2006, pp. 109-113. doi:10.1016/j.physleta.2006.07.065

[18] R. A. Van Gorder and K. Vajravelu, "Analytic and Numerical Solutions to the Lane-Emden Equation," Physics letter A, Vol. 372, No. 39, 2008, pp. 6060-6065. doi:10.1016/j.physleta.2008.08.002

[19] S. Liang and D. J. Jeffrey, "Comparison of Homotopy Analysis Method and Homotopy Perturbation Method through an Evolution Equation," Communications in Nonlinear Science and Numerical Simulation, Vol. 14, No. 12, 2009, pp. 4057-4064. doi:10.1016/j.cnsns.2009.02.016

[20] J. H. He, "Variational Iteration Method-Some Recent
Results and New Interpretations," Journal of Computational and Applied Mathematics, Vol. 207, No. 1, 2007, pp. 3-17. doi:10.1016/j.cam.2006.07.009

[21] S. J. Liao, "A Uniformly Valid Analytic Solution of Two-Dimensional Viscous Flow over a Semi-Infinite Flat Plate," Journal of Fluid Mechanics, Vol. 385, 1999, pp. 101-128.

[22] S. J. Liao and I. Pop, "Explicit Analytic Solution for Similarity Boundary Layer Equations," International Journal of Heat and Mass Transfer, Vol. 47, No. 1, 2004, pp. 75-85. doi:10.1016/S0017-9310(03)00405-8

[23] J. H. He, "Some Asymptotic Methods for Strongly Nonlinear Equations," International Journal of Modern Physics B, Vol. 20, No. 10, 2006, pp. 1141-1199. doi: $10.1142 / \mathrm{S} 0217979206033796$ 